\title{
$\delta$-Doped MOS Ge/Si quantum dot/well infrared photodetector
}

\author{
C.-H. Lin ${ }^{\text {a }}$, C.-Y. Yu ${ }^{\text {a }}$, P.-S. Kuo ${ }^{\text {a }}$, C.-C. Chang ${ }^{\text {a }}$, T.-H. Guo ${ }^{\text {a }, \text { C.W. Liu }}{ }^{\text {a,b,* }}$ \\ ${ }^{a}$ Department of Electrical Engineering and Graduate Institute of Electronics Engineering, National Taiwan University, Taipei, Taiwan, ROC \\ ${ }^{\mathrm{b}}$ ERSO/ITRI, Hsinchu, Taiwan, ROC
}

Available online 28 November 2005

\begin{abstract}
Metal-oxide-semiconductor tunneling diodes can be used as photodetectors at the inversion bias. To increase the responsivity, $\delta$-doping is used in the quantum dot infrared photodetectors (QDIPs) and quantum well infrared detectors (QWIPs). The peak responsivity of the quantum dot and quantum well infrared photodetector at $15 \mathrm{~K}$ is found to be $0.03 \mathrm{~mA} / \mathrm{W}$ and $1.3 \mathrm{~mA} / \mathrm{W}$, respectively, at a gate voltage of $1 \mathrm{~V}$. The higher responsivity of the QWIP is probably due to the continuous two-dimensional density of states and smaller transition energy as compared to the QDIP. The QD photoresponse in the peak wavelength range 3.5-5 $\mu \mathrm{m}$ can be measured up to $100 \mathrm{~K}$, while that for QW 3-7 $\mu \mathrm{m}$ can only be detected up to $60 \mathrm{~K}$. A higher dark current due to the lower transition energy in the QWIP limits its operating temperature as compared to the QDIP.
\end{abstract}

(C) 2005 Elsevier B.V. All rights reserved.

Keywords: MOS; Silicon; Germanium

\section{Introduction}

Advanced Si-heterostructure technology can increase the functionality of $\mathrm{Si}$ chips with potential applications in optoelectronic devices [1-4]. An ultra-thin insulator in metal-insulator-semiconductor (MIS) structure allows a significant tunneling gate current, which can be used in the photodetectors. Note that a large gate current is not desired in ultra-large scale integration circuit applications.

Mid- and long-wavelength quantum dot/well infrared photodetectors using intraband transitions are attractive in the military, medical, astronomical and other applications [5]. Due to the requirement of a precise control of heterojunction abruptness, most quantum dot/well infrared photodetectors (QDIPs/QWIPs) are grown by Molecular Beam Epitaxy (MBE). In this paper, we have successfully fabricated QDIPs/ QWIPs with good performances by Ultrahigh Vacuum Chemical Vapor Deposition (UHVCVD). Schematic structure is shown in Fig. 1.

\footnotetext{
* Corresponding author. Department of Electrical Engineering and Graduate Institute of Electronics Engineering, National Taiwan University, Taipei, Taiwan, ROC. Tel.: +886 2 23635251x515; fax: +886223638247.

E-mail address: chee@cc.ee.ntu.edu.tw (C.W. Liu).
}

\section{2. $\delta$-doped quantum dot infrared photodetector}

A MOS structure at the inversion bias can significantly reduce the dark current due to the tunneling oxide. Fig. 2 shows that a 5-period of $\mathrm{Ge} / \mathrm{Si}$ quantum dot infrared photodetector (QDIP) in metal-semiconductor (MS) structure has larger dark current than that in metal-oxide-semiconductor (MOS) structure without $\delta$-doping introduced. The hole excited by an infrared exposure in $\mathrm{Ge} / \mathrm{Si}$ quantum dots (quantum wells) can transport into the back electrode by relaxation in the p-type semiconductor (the inset of Fig. 3). The $\delta$-doping in the quantum dots (quantum wells) can increase the responsivity due to the increase of hole concentration inside the dots (wells). The valence band offset between $\mathrm{Si}$ and $\mathrm{Ge}(\mathrm{SiGe})$ forms discrete quantum states in the Ge quantum dots and two-dimensional density of state in the $\mathrm{Si}_{0.7} \mathrm{Ge}_{0.3}$ quantum wells [6]. The hole transition energy is determined by the band structure of the quantum dots (quantum wells).

A 20-period Ge/Si quantum dots (QDs) with $3 \mathrm{~nm}$ wetting layer grown by UHVCVD was fabricated into MOS tunneling diodes [7] with a low-temperature $\left(50{ }^{\circ} \mathrm{C}\right)$ liquid phase deposition (LPD) oxide (Fig. 1) [8]. A boron concentration of $10^{19} \mathrm{~cm}^{-3}$ was introduced during the growth of Ge quantum dots. Boron incorporation occurred only for the middle onetenth of deposition time of the Ge layer. Ge quantum dot layers 


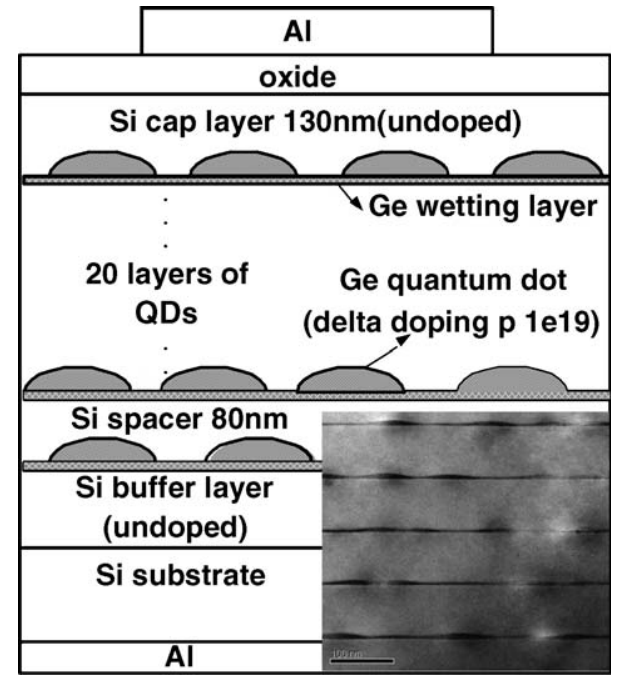

Fig. 1. The structure of MOS Ge/Si QDIP. Twenty-layer Ge quantum dots with $\delta$-doping were prepared by UHVCVD. The inset shows the TEM photograph of the quantum dot structure. The Si spacer is $80 \mathrm{~nm}$ in thickness.

were separated by $80 \mathrm{~nm} \mathrm{Si} \mathrm{spacer} \mathrm{layer.} \mathrm{The} \mathrm{cross-sectional}$ TEM photograph of the QD structure is shown in the inset of Fig. 1. Al was deposited on the oxide to form the gate electrode with an area of $3 \times 10^{-2} \mathrm{~cm}^{2}$.

When the Si spacer is smaller than the correlation length, the grown Ge QDs can be self-assembled in a row vertically [9]. However, in this study, the Si spacer is $80 \mathrm{~nm}$ thick, which is too high to have the strain field coupling between quantum dot layers. The Ge dot is not necessarily aligned vertically in a row in our samples, as indicated in the TEM micrograph in Fig. 1.

Fig. 3 shows the $I-V$ characteristics of the NMOS QDIP at different temperatures. At inversion bias, one component of the dark current of MOS tunneling diode is dominated by the thermal generation of electron-hole pairs from the valence to the conduction band through the defects in the depletion region and at the $\mathrm{Si} / \mathrm{SiO}_{2}$ interface (the inset of Fig. 3). Thermally generated electron-hole pairs reduce as the operating temperature decreases. The other component of the dark current arises from the holes thermally excited from the quantum dots to the valence band edge (the inset of Fig.



Fig. 2. Comparison of dark current between MOS and MS structures.

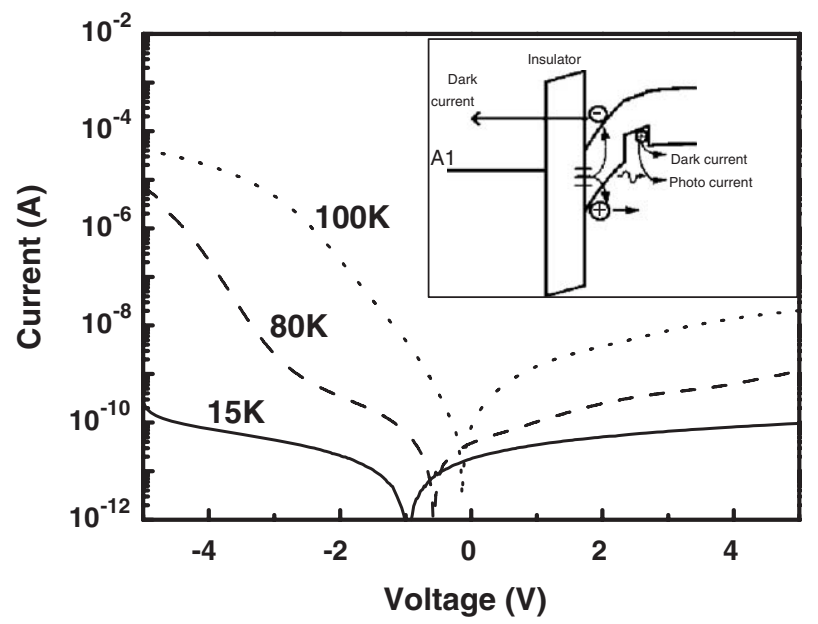

Fig. 3. Dark current of a MOS Ge/Si QDIP at different temperatures. The inset shows the band diagram of a NMOS Ge/Si QDIP at inversion bias. The confined holes can be excited under infrared exposures.

3). The dark current at the inversion bias decreases as the operating temperature reduces. At accumulation bias, the electron tunnels from the $\mathrm{Al}$ gate to the $\mathrm{p}-\mathrm{Si}$, and the accumulated holes at oxide/Si interface also tunnel to the $\mathrm{Al}$ gate electrode. Both tunneling mechanisms are related to the trap-assisted tunneling (Frenkel-Poole), and decrease with the lowering of temperature.

Under infrared exposures at an inversion bias, the confined holes can be excited and contribute to photocurrent. The spectral response is measured by Fourier transform infrared (FTIR) spectrometer (Perkin-Elmer Spectrum 2000) coupled with a cryostat [APD cryogenics] and a SR570 current preamplifier. The incident light is unpolarized and the devices are under normal incidence detection mode.

At $15 \mathrm{~K}$, a broad $3.5-5 \mu \mathrm{m}$ peak, originated from the intraband transition in the quantum dot, is observed at gate voltages of zero and $1 \mathrm{~V}$ with a peak responsivity of $0.03 \mathrm{~mA} /$ $\mathrm{W}$ at $1 \mathrm{~V}$ (Fig. 4). This intraband transition at $3.5-5 \mu \mathrm{m}$ is different from the transition $(3-10 \mu \mathrm{m})$ in the undoped devices, probably due to another intraband transition resulting from more holes at higher energy level. At $20 \mathrm{~K}$, the responsivity of undoped-QDIPs is $0.004 \mathrm{~mA} / \mathrm{W}$ at $6.6-\mu \mathrm{m}$ peak [10]. The $\delta$ doping is expected to contribute hole concentration in the doped devices. The dark current at $80 \mathrm{~K}$ for the $\delta$-doped device

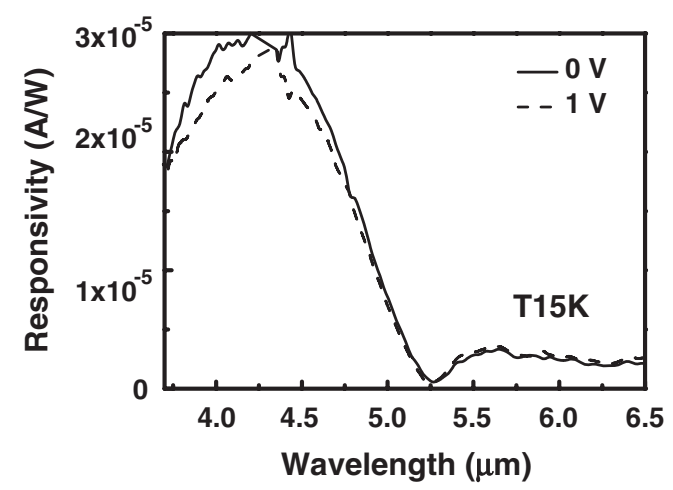

Fig. 4. Spectral response of a QD device at different gate voltages. 


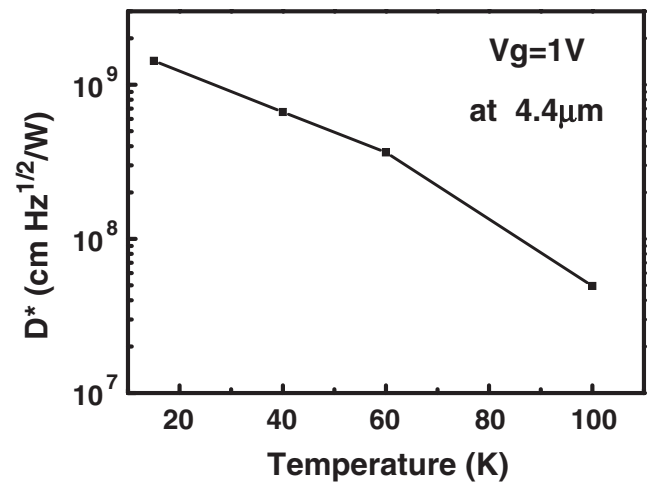

Fig. 5. The detectivity of the $3.5-5 \mu \mathrm{m}$ peak vs. temperature at a gate voltage of $1 \mathrm{~V}$ for the QDIP.

is $\sim 10^{-9} \mathrm{~A}$, which is 10 times larger than that of undoped QDIPs, because more holes can be thermally excited from the doped quantum dots. The operating temperature is also reduced due to the larger dark current. There is a trade-off between the operating temperature and responsivity.

The normalized detectivity $D^{*}$ is defined as:

$D^{*}=\frac{\sqrt{A \Delta f}}{\mathrm{NEP}}=\frac{\sqrt{A \Delta f}}{i_{\mathrm{n}} / R}$

where $A$ is the detector area $\left(3 \times 10^{-2} \mathrm{~cm}^{2}\right), \Delta f$ is the equivalent bandwidth of the electronic system. The noise equivalent power (NEP) is defined as $i_{n} / R$, where $i_{\mathrm{n}}$ is the current noise and $R$ is the responsivity. The current noise is limited by the dark current and can be approximated as the shot noise $\left(2 e I_{\mathrm{d}} \Delta f\right)^{1 / 2}$, where $I_{\mathrm{d}}$ is the measured dark current. Therefore, $D^{*}$ can be simplified to

$D^{*}=\frac{\sqrt{A} R}{\sqrt{2 e I_{\mathrm{d}}}}$.

Fig. 5 shows the detectivity at different temperatures at $1 \mathrm{~V}$ bias. The peak detectivity is found to be $10^{9} \mathrm{~cm}-\mathrm{Hz}^{1 / 2} / \mathrm{W}$ for the $3.5-5 \mu \mathrm{m}$ peak. The detectivity decreases with the increase of operating temperature due to the rise of the dark current. For

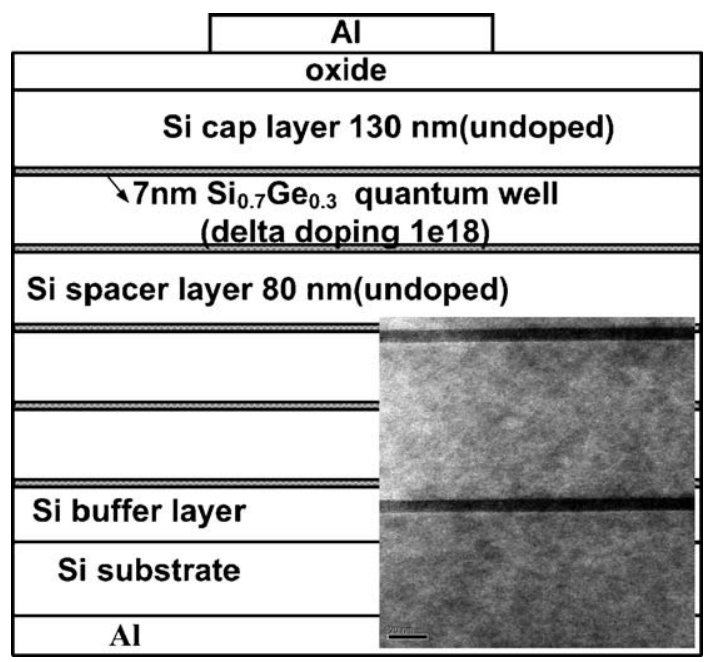

Fig. 6. The structure of the MOS $\mathrm{Si}_{0.7} \mathrm{Ge}_{0.3}$ QWIP. The inset shows the TEM photograph of the quantum well structure.

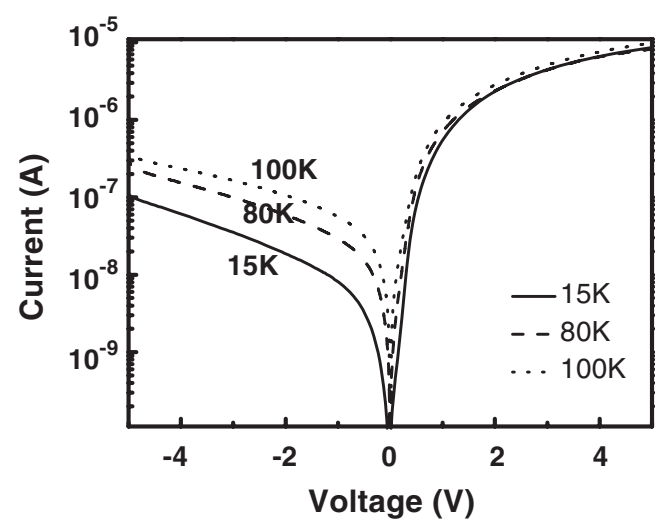

Fig. 7. Dark current of MOS QWIP devices as a function of operating temperature.

comparison, the detectivity of III-V based-ODIP (InGaAs/ AlGaAs quantum well on GaAs substrates) is $4 \times 10^{10} \mathrm{~cm}-\mathrm{Hz}^{1 /}$ ${ }^{2} / \mathrm{W}$ for $4.5 \mu \mathrm{m}$ peak at $95 \mathrm{~K}[11]$.

\section{3. $\delta$-Doped quantum well infrared photodetector}

The $\delta$-doping was also introduced in the $\operatorname{MOS} \mathrm{Si}_{0.7} \mathrm{Ge}_{0.3}$ quantum well infrared photodetector (QWIP). The structure is similar to the QD device, but the QDs are replaced by 5 layers of 7-nm-thick $\mathrm{Si}_{0.7} \mathrm{Ge}_{0.3}$ QWs with $80 \mathrm{~nm}$ spacers (Fig. 6). Boron with the concentration of $10^{18} \mathrm{~cm}^{-3}$ was introduced during the growth of $\mathrm{Si}_{0.7} \mathrm{Ge}_{0.3}$ quantum wells and the same incorporation time with the $\delta$-doped QDIP mentioned above. The TEM photograph of the QW structure is shown in the inset of Fig. 6.

In comparison, the dark current of the $\delta$-doped QW device (Fig. 7) is larger than that of the $\delta$-doped QD device at low temperatures. Due to the smaller confinement energy for holes in SiGe QW, holes can be easily thermally excited and contribute to the dark current. The continuum of the twodimensional density of states in quantum wells also assists the thermal excitation of holes out of the quantum well, as compared to the discrete energy states in quantum dots.

The spectral response of $\delta$-doped QWIP at $15 \mathrm{~K}$ for different gate voltages is shown in Fig. 8. Due to the smaller



Fig. 8. Spectral response of a QW device at different gate voltages. For $V_{\mathrm{G}}=1$ $\mathrm{V}$, the peak responsivity at $15 \mathrm{~K}$ is $1.3 \mathrm{~mA} / \mathrm{W}$. 


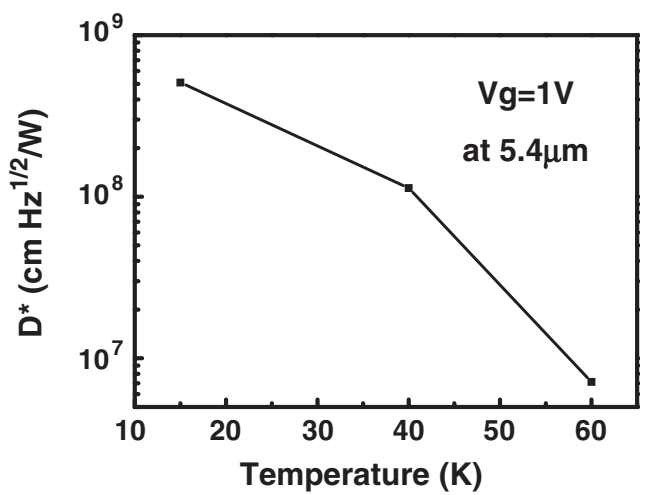

Fig. 9. The detectivity of the $3-7 \mu \mathrm{m}$ peak vs. temperature at $V_{\mathrm{G}}=1 \mathrm{~V}$ for the QWIP. The response becomes weak beyond $60 \mathrm{~K}$.

confinement energy of the SiGe QW device, the absorption wavelength shifts to longer wavelength $(3-7 \mu \mathrm{m})$. For $V_{\mathrm{G}}=1$ $\mathrm{V}$, the peak responsivity at $15 \mathrm{~K}$ is $1.3 \mathrm{~mA} / \mathrm{W}$. The SiGe $\delta$ doped QW detector has larger responsivity than that of the $\delta$ doped QD detector. Photo-excited holes in the detectors may be trapped by subsequent layers of quantum dots/quantum wells. Trapped holes in the QW device are more easily thermally excited due to a small transition energy. This may lead to the larger responsivity as compared to the $\delta$-doped QD device, but the exact origin is still under investigation.

The detectivity at different temperatures at $1 \mathrm{~V}$ bias is plotted in Fig. 9. The peak detectivity is $5 \times 10^{8} \mathrm{~cm}-\mathrm{Hz}^{1 / 2} / \mathrm{W}$ at $5.4 \mu \mathrm{m}$ and decreases with increasing temperature. Due to the larger dark current, the operating temperature of the QW device is lower than that of the QD device.

\section{Conclusion}

MOS-based $\mathrm{Ge} / \mathrm{Si}$ quantum dot/well photodetectors are demonstrated by introducing $\delta$-doping using UHVCVD. For QDIP, a peak in wavelength range $3.5-5 \mu \mathrm{m}$, which originates from the intraband transition in the quantum dot, is observed.
At $15 \mathrm{~K}$, the peak $3.5-5 \mu \mathrm{m}$ responsivity is $0.03 \mathrm{~mA} / \mathrm{W}$ at $1 \mathrm{~V}$, and the operating temperature of the device is up to $100 \mathrm{~K}$. A higher peak responsivity is observed for the $\delta$-doped QWIP with the highest value reaching $1.3 \mathrm{~mA} / \mathrm{W}$ at $1 \mathrm{~V}$. The absorption region for the devices is 3 to $7 \mu \mathrm{m}$. However, due to larger dark current, the operating temperature of the QW device is only up to $60 \mathrm{~K}$.

\section{Acknowledgment}

This work is supported by U.S. Air Force Office of Scientific Research/AOARD under contract no. FA520904P0441, and National Science Council, Taiwan.

\section{References}

[1] Min Yang, Jeremy Schaub, Dennis Rogers, Mark Ritter, Ken Rim, Jeffrey Welser, Byeongju Park, International Electron Device Meeting, 2001 , p. 547.

[2] J., Kolodzey, T.N., Adam, R.T., Troeeger, P.-C., Lv, S.K., Ray, G., Looney, A., Rosen, M.S., Kagan, Irina N., Yassievich, "The Design and Operation of TeraHertz Sources Based on Silicon Germanium Alloys," Topical Meeting on Silicon Monolithic Integrated Circuits in RF Systems, 9-11th April, 2003, Grainau, Germany.

[3] A.I. Yakimov, A.V. Dvurechenskii, A.I. Nikiforov, Yu. Yu. Proskuryakov, J. Appl. Phys. 89 (2001) 5676.

[4] R. People, J.C. Bean, C.G. Bethea, S.K. Sputz, L.J. Peticolas, Appl. Phys. Lett. 61 (1992) 1122.

[5] A. Rogalski, J. Appl. Phys. 93 (2003) 4355.

[6] R.P.G. Karunasiri, J.S. Park, Y.J. Mii, K.L. Wang, Appl. Phys. Lett. 57 (1990) 2585.

[7] C.W. Liu, M.H. Lee, C.F. Lin, I.C. Lin, W.T. Liu, H.H. Lin, Technical Digest, International Electron Device Meeting, Washington, D.C., 1999, p. 749.

[8] B.-C. Hsu, S.T. Chang, C.-R. Shie, C.-C. Lai, P.S. Chen, C.W. Liu, International Electron Device Meeting, 2002, p. 91.

[9] O.G. Schmidt, K. Eberl, Phys. Rev., B. 61 (2000) 13721.

[10] B.-C. Hsu, C.-H. Lin, P.-S. Kuo, S.T. Chang, P.S. Chen, C.W. Liu, J.-H. Lu, C.H. Kuan, IEEE Electron Device Lett. 25 (2004) 544.

[11] A. Fiore, E. Rosencher, P. Bois, J. Nagle, N. Laurent, Appl. Phys. Lett. 64 (1994) 478. 